\title{
Introduction of Local Spatial Constraints and Local Similarity Estimation in Possibilistic c-Means Algorithm for Remotely Sensed Imagery
}

\author{
Abhishek Singh*, Anil Kumar \\ Indian Institute of Remote Sensing (IIRS), Indian Space Research Organisation (ISRO), Dehradun, 248001, \\ India \\ E-mail: abhisheksingh2441@gmail.com (Corresponding author); anil@iirs.gov.in
}

Received: 5 March 2019; Accepted: 25 March 2019; Available online: 10 June 2019

\begin{abstract}
This paper presents a unique Possibilistic c-Means with constraints (PCM-S) with Adaptive Possibilistic Local Information c-Means (ADPLICM) in a supervised way by incorporating local information through local spatial constraints and local similarity measures in Possibilistic c-Means Algorithm. PCM-S with ADPLICM overcome the limitations of the known Possibilistic c-Means (PCM) and Possibilistic c-Means with constraints (PCM-S) algorithms. The major contribution of proposed algorithm to ensure the noise resistance in the presence of random salt \& pepper noise. The effectiveness of proposed algorithm has been analysed on random "salt and pepper" noise added on original dataset and Root Mean Square Error (RMSE) has been calculated between original dataset and noisy dataset. It has been observed that PCM-S with ADPLICM is effective in minimizing noise during supervised classification by introducing local convolution.

Keywords: Possibilistic c-MEANS (PCM); Possibilistic c-Means with constraints (PCM-S); Local similarity measures; Pixel spatial attraction mode; Mean membership difference (MMD); Root mean square error (RMSE).
\end{abstract}

\section{Introduction}

Remote Sensing technology has been widely used to obtain useful information for extraction and discrimination of land cover by assigning a class label to each pixel in a digital image. Lillesand et al [1] have mentioned digital image classification as a statistical technique to classify image into various desired classes. Digital image classification plays a key role in extracting land cover information using hard and fuzzy classification approach. Each pixel is assumed to be pure in case of hard classification and is classified to one class but in case of fuzzy classification mixed pixel problem exist and each pixel is assigned to multiple class memberships. Digital Image classification can be done in supervised as well as in unsupervised way.

Bezdek [2] presented Fuzzy c-Means (FCM) algorithm with the thought of fuzzy sets to solve mixed pixel problem which was previously introduced by Zadeh [3]. Zadeh's idea was to assign a particular sample or pixel to more than one class with the help of a membership grade varying between 0 and 1. Krishnapuram et.al [4] presented a possibilistic fuzzy approach to interpret the membership value of pixel as a degree of possibility and later Krishnapuram et al [5] addressed the drawbacks associated with the constrained memberships used in FCM algorithm. Wu et.al [6] proposed an alternative $c$-means clustering algorithm to replace the Euclidean norm in cmeans clustering procedures on the basis of the robust statistic and the influence function.

Li et al [7] revised the objective function of PCM and introduced MPCM which is less sensitive to noise, fit the clusters which are close to one another and it is one of the fast clustering algorithm as compared to FCM and PCM. Kumar et al [8] compared the accuracy of FCM against PCM and found that, PCM with Euclidean norm has the highest overall accuracy among them. Chawla [9] analysed the performance of PCM and] found to be better than FCM because PCM was able to surpass the effect of hyper-line constant found in FCM. In the context of remote sensing image analysis, MRF was used for image restoration [10], texture classification [11], image segmentation [12] etc. The local contextual information helps to establish some relationship among the neighbouring pixels [13].

Ahmed et al [14] proposed Fuzzy c-Means with constraints (FCM-S) in which objective function of FCM is modified in order to minimize the limitation of FCM by allowing the label of pixel to be influenced by labels of neighbors. Chen et al [15] proposed FCM_S1 and FCM_S2, two variants of FCM_S algorithm in order to resolve the problem of FCM_S by introducing mean and median filtered image respectively to replace the neighborhood term of FCM_S. In all these FCM based algorithms needs a crucial parameter to control the trade-off between the robustness to noise and the effectiveness of preserving the image details. Krinidis et al [16] introduced FLICM (Fuzzy Local Information c-Means) algorithm in order to overcome the problem in FCM-S, FCM-S1 and FCM- 
S2. Zhang et al [17] presented ADFLICM (Adaptive Fuzzy Local Information c-Means) algorithm to address the limitation of FLICM.

\section{Mathematical concept of preliminary algorithm}

Suppose an image $X=\left\{x_{1}, x_{2}, x_{3}, \ldots x_{1} \ldots \ldots x_{N}\right\}, x \in R$, is a dataset in $n$-dimensional vector space and $N$ is the number of feature vectors.

\subsection{Possibilistic c-Means (PCM) algorithm}

In the PCM algorithm, each cluster is independent of the other clusters [5]. The objective function can be written as in eq. (1):

$$
J_{m}=\sum_{i=1}^{N} \sum_{j=1}^{C}\left(u_{j i}\right)^{m}\left\|x_{i}-v_{j}\right\|^{2}+\sum_{i=1}^{N} \eta_{i} \sum_{j=1}^{C}\left(1-u_{j i}\right)^{m}
$$

The membership update equation in the PCM is

$$
u_{j i}(n+1)=\frac{1}{1+\sum_{i=1}^{N}\left[\frac{\left\|x_{i}-v_{j}\right\|^{2}}{\eta_{i}}\right]^{\frac{1}{m-1}}}
$$

In equation (2), $\boldsymbol{v}_{\boldsymbol{j}}$ represents the prototype associated with class C, and $\eta_{i}$ is the "bandwidth", "resolution" or "scale" parameter which controls the shape and size of the class as in eq. (3);

$$
\eta_{i}=\frac{\sum_{i=1}^{N}\left(u_{j i}\right)^{m}\left\|x_{i}-v_{j}\right\|^{2}}{\sum_{i=1}^{N}\left(u_{j i}\right)^{m}}
$$

where, $\mathrm{N}$ is the total number of pixels in the image, $i$ represents a pixel and it varies from 1 to $\mathrm{N}$.

In equation (1), first term controls the distance between the feature vectors i.e. the pixels and their prototypes to be low as possible and the second term, demands $u_{j i}$ to be large as possible, by controlling the onset of trivial solution. The objective function, $J_{m}$ in equation (1) and membership value, $u_{j i}$ in equation (2) satisfies the following condition given as eq. (4), (5) and (6):

$$
\begin{aligned}
& u_{j i} \in[0,1], \forall i, j \\
& 0<\sum_{i=1}^{N} u_{j i} \leq 1, \forall i \\
& \max _{j} u_{j i}>0, \forall i
\end{aligned}
$$

PCM Algorithm includes the steps as follows:

Step 1: Assign mean values for each class,

Step 2: Assign the value of degree of fuzziness $m>1$,

Step 3: Compute the regularization parameter $\eta_{j}$ from eq. (3),

Step 4: Calculate the membership matrix $u_{j i}$ from eq. (2),

Step 5: Assign final class to each pixel.

\subsection{Possibilistic c-Means with constraints (PCM-S) algorithm}

PCM-S has been introduced a novel term that enables the labelling of a pixel to be effected by labels of its neighbors.

The neighborhood effect act as an allocator and drives the solution toward piecewise-homogeneous labelling. The modified objective function of PCM-S is defined in eq. (7) Singh et al [18].

$$
J_{m}=\sum_{i=1}^{N} \sum_{j=1}^{C}\left(u_{j i}\right)^{m}\left\|x_{i}-v_{j}\right\|^{2}+\sum_{i=1}^{N} \eta_{i} \sum_{j=1}^{C}\left(1-u_{j i}\right)^{m}+\frac{\alpha}{N_{R}} \sum_{i=1}^{N} \sum_{j=1}^{C}\left(u_{j i}\right)^{m} \sum_{r \in N_{i}}\left\|x_{r}-v_{j}\right\|^{2}
$$

where, $x_{j}$ is the gray level value of $\mathrm{i}^{\text {th }}$ pixel, $\mathrm{N}$ is the total number of pixels, $v_{j}$ is the prototype value of the $\mathrm{j}^{\text {th }}$ center, $u_{j i}$ represents the fuzzy membership of $\mathrm{i}^{\text {th }}$ pixel with respect to class $\mathrm{j}, \mathrm{N}_{\mathrm{R}}$ is the cardinality, $x_{\mathrm{r}}$ represents the neighbor of $x_{1}$. The parameter is $\alpha$ used to supervise the effect of the neighbors term . 
The calculation of c membership matrix is performed as follows in eq. (8)

$$
u_{j i}=\frac{\left[\frac{\left\|x_{i}-v_{j}\right\|^{2}}{\eta_{i}}+\frac{\alpha}{N_{R}} \sum_{\substack{r \neq N_{i} \\ r \neq i}} \frac{\left\|x_{r}-v_{j}\right\|^{2}}{\eta_{i}}\right]^{\frac{1}{m-1}}}{1+\sum_{j=1}^{C}\left[\frac{\left\|x_{i}-v_{j}\right\|^{2}}{\eta_{i}}+\frac{\alpha}{N_{R}} \sum_{\substack{r \in N_{i} \\ r \neq i}} \frac{\left\|x_{r}-v_{j}\right\|^{2}}{\eta_{i}}\right]}
$$

and it satisfies the following condition given as eq. (4), (5) and (6).

In equation (8) $\frac{1}{N_{R}} \sum_{r \in N_{i}} x_{r}$ in the numerator is a neighbour average grey level value around $\boldsymbol{x}_{\boldsymbol{i}}$ within a window. PCM-S Algorithm includes the steps as follows:

Step 1: Assign mean values for each class,

Step 2: Assign the value of degree of fuzziness $\mathrm{m}>1$, parameter ' $\alpha$ ' and convolution window size $\left(N_{R}\right)$,

Step 3: Compute the regularization parameter $\eta_{i}$ from eq. (3),

Step 4: Compute the membership matrix $u_{j i}$ from eq. (8),

Step 5: Assign final class to each pixel.

\section{Mathematical concept of PCM-S with ADPLICM algorithm}

Zhang et al [17] have used Local Similarity Measure based on Pixel Spatial Attraction Model in Adaptive Fuzzy Local Information $c$-means which adaptively determines the weighting factors for neighboring, the same way we have been applied for PCM-S with ADPLICM based fuzzy classifier.

Pixel Spatial Attraction between two pixels can be defined as in eq. (9):

$$
S A_{i j(k)}=\frac{u_{k i} \times u_{k j}}{d_{j i}^{2}}
$$

where $d_{j i}$ is a spatial Euclidean distance between two pixels i and j. Further Zhang et al [17] proposed similarity measures to provide a well suited balance between the insensitiveness to noise and effectiveness of preserving the image details. Based on the concept of local spatial correlation between pixels in image, a novel local similarity measure $S_{\text {ir }}$ has been introduced to incorporate both local information which is defined as in eq. (10):

$$
\begin{aligned}
& S_{i r}= \begin{cases}S A_{i r}, & \mathrm{i} \neq \mathrm{r} \\
0, & i=r\end{cases} \\
& r \in 0<\left(x_{i}-x_{r}\right)^{2}+\left(y_{i}-y_{r}\right)^{2} \leq 2^{L-1}
\end{aligned}
$$

where, $\mathrm{i}$ depicts the center pixel of local window, $\mathrm{r}^{\text {th }}$ pixel is the neighborhood pixel, $\left(x_{i}-x_{r}\right)$ and $\left(y_{i}-y_{r}\right)$ denote the coordinates of pixel $\mathrm{i}$ and $\mathrm{r}$ respectively. In this paper, ADPLICM is introduced for supervised classification and to incorporate local spatial and gray level information into objective function of PCM. The objective function of PCM-S with ADPLICM is represented as in eq. (12):

$$
\begin{gathered}
J_{m}=\sum_{i=1}^{N} \sum_{k=1}^{C}\left(u_{k i}\right)^{m}\left\|x_{i}-v_{k}\right\|^{2}+\sum_{i=1}^{N} \eta_{i} \sum_{k=1}^{C}\left(1-u_{i k}\right)^{m}+\frac{\alpha}{N_{R}} \sum_{i=1}^{N} \sum_{j=1}^{C}\left(u_{j i}\right)^{m} \sum_{r \in N_{i}}\left\|x_{r}-v_{j}\right\|^{2}+ \\
\sum_{i=1}^{N} \sum_{k=1}^{C}\left(u_{k i}\right)^{m} \frac{1}{N_{R}} \sum_{\substack{r \in N_{i} \\
r \neq i}}\left(1-S_{i r}\right)\left\|x_{r}-v_{k}\right\|^{2}
\end{gathered}
$$

The calculation of membership partition matrix is performed as follows in eq. (13)

$$
u_{k i}=\frac{1}{1+\sum_{j=1}^{c}\left[\frac{\frac{\left\|x_{i}-v_{k}\right\|^{2}}{\eta_{i}}+\frac{\alpha}{N_{R}} \sum_{\substack{r \in N_{i} \\ r \neq i}}\left(1-S_{i r} r \frac{\left\|x_{r}-v_{k}\right\|^{2}}{\eta_{i}}\right.}{\frac{\left\|x_{i}-v_{j}\right\|^{2}}{\eta_{i}}+\frac{\alpha}{N_{R}} \sum_{\substack{r \in N_{i} \\ r \neq i}}\left(1-s_{i r}\right) \frac{\left\|x_{r}-v_{j}\right\|^{2}}{\eta_{i}}}\right]^{\frac{1}{m-1}}}
$$

and it satisfies the following condition given as eq. (4), (5) and (6).

The flowchart of (PCM-S with ADPLICM) algorithm is shown in Fig. 1. The implementation includes following four steps; 
Step 1: Assign mean values for each class.

Step 2: Assign the value of fuzzification factor $m$ and the local window.

Step 3: Calculate regularization parameter $\eta_{i}$ from eq. (3) and local similarity measures $S_{\text {ir }}$ from equation (10),

Step 4: Calculate class centers and final membership matrix $u_{k i}$ from eq. (13),

Step 5: Assign final class to each pixel.

In this paper, we are introducing the same concept by incorporating local information in characterizing the spatial correlation between pixels in the image. Zhang et al [17] said: “ For two pixels i and j, their attraction w.r.t. the $\mathrm{k}^{\text {th }}$ class is proportional to their membership value $u_{k i}$ and $u_{k j}$ and inversely proportional to the square of the spatial distance between the two pixels”.

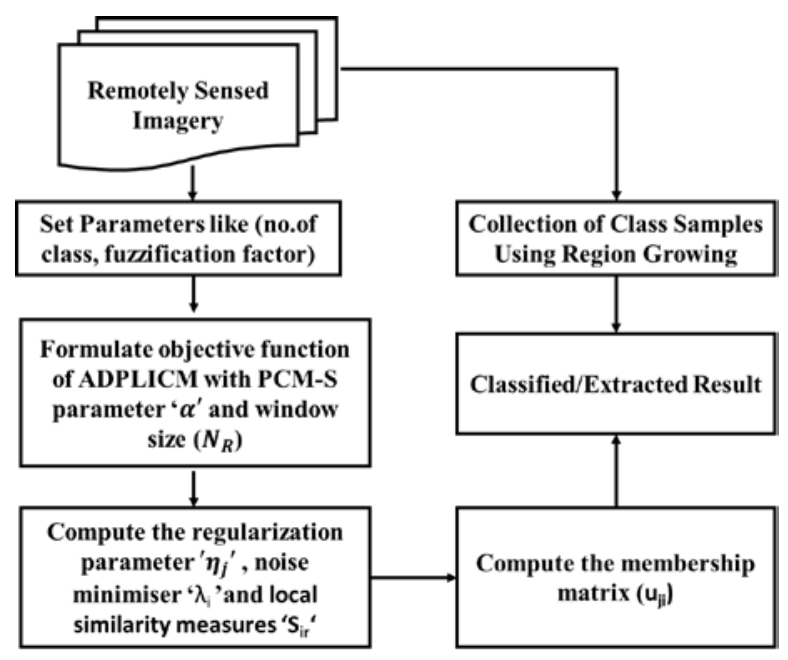

Fig.1. Flowchart of the proposed PCM-S with ADPLICM algorithm

\section{Experimental results}

In this section results of supervised PCM, PCM-S and PCM-S with ADPLICM algorithms has been compared. Base classifier PCM and PCM-S has been optimized using mean membership difference (MMD). MMD is an independent approach for the stability of concerned class by calculating the mean difference of membership value of concerned class and other classes of pure pixel [18]. In Fig. 2, effect of variation of fuzzification factor (m) has been compared for Wheat in PCM and PCM-S algorithms. Fuzzification factor (m) has been varied with a difference of 0.2 in case of FORMOSAT-2 satellite imagery. The value of fuzzification factor (m) at which MMD value is high indicates the optimization point of base classifier.

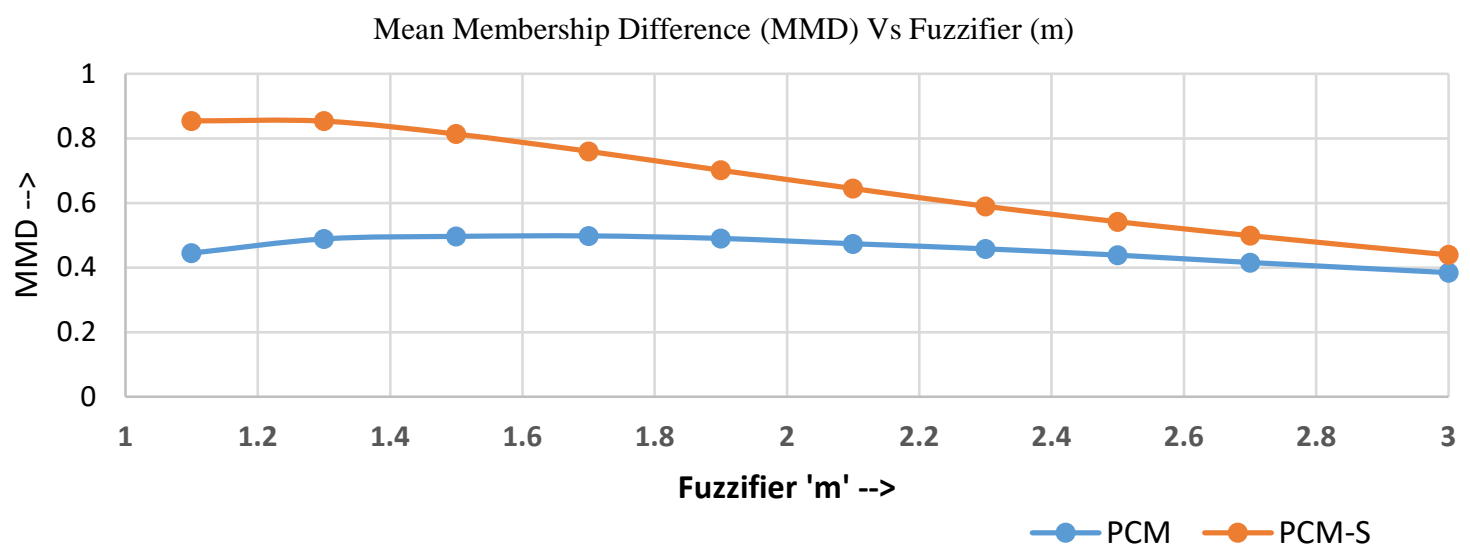

Fig. 2. Mean Membership Difference Plot: Wheat Class

In this experiment $8 \mathrm{~m}$ resolution multispectral Formosat-2 Image (1333 x 1372 pixels) is tested in which $1 \%$ Salt \& Pepper random noise were added separately in the original image (1333 x 1372 pixels). Original Image (1333 x 1372 pixels) and Noisy Image (1333 x 1372 pixels) have been classified using PCM, PCM-S and PCM-S 
with ADPLICM in supervised way. The difference between Original classified image and Noisy classified image have been calculated using RMSE. RMSE is basically calculated by measuring the difference between original classified image and noisy classified image. PCM-S with ADPLICM algorithm is removed the noise effectively by incorporating local information. Fig. 3 (a) shows the Standard FCC of Formosat-2 image (1333 x 1372 pixels), Haridwar Site, Uttarakhand (2955’36.94” N, 786’ 16.37” E to 2948’37.30” N, 78¹4’55.13” E). Fig. 3 (b), (c) and (d) shows classified image of Wheat Class through PCM, PCM-S and PCM-S with ADPLICM algorithm respectively.

Table1 displays the quantitative results. As mentioned in Table 1, PCM-S with ADPLICM has less RMSE RMSE means the effect of Noisy immunity is high. The RMSE of PCM, PCM-S and PCM-S with ADPLICM are $0.081,0.069$ and 0.066 respectively.

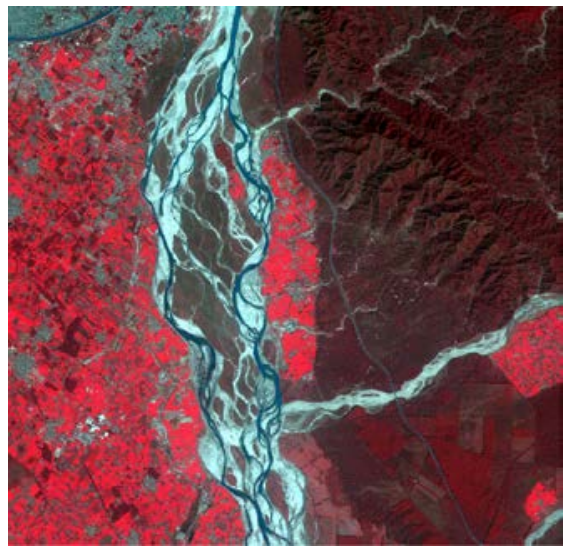

(a)

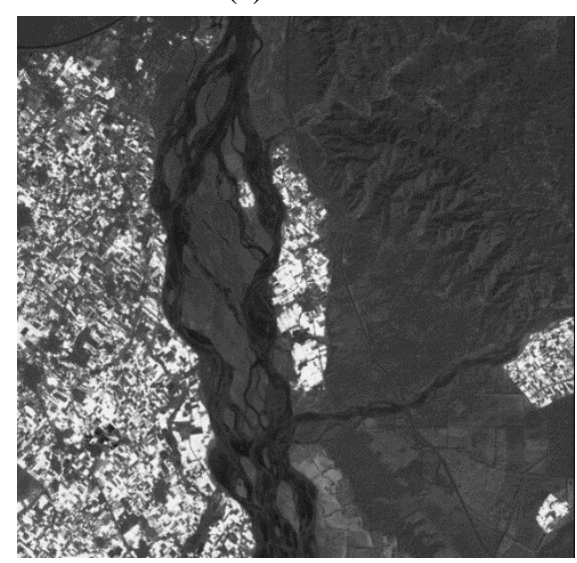

(c)

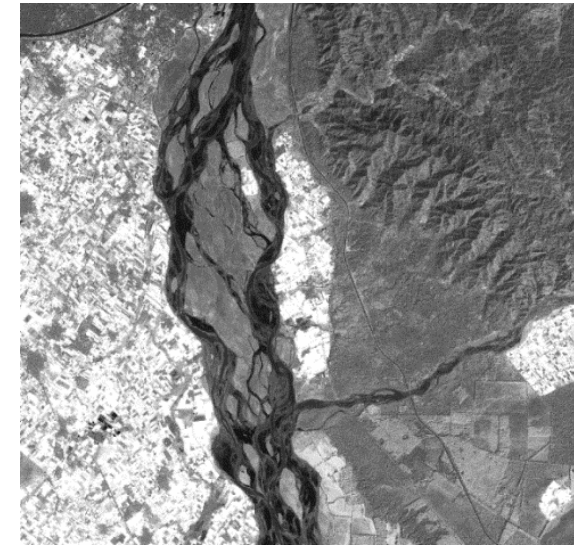

(b)

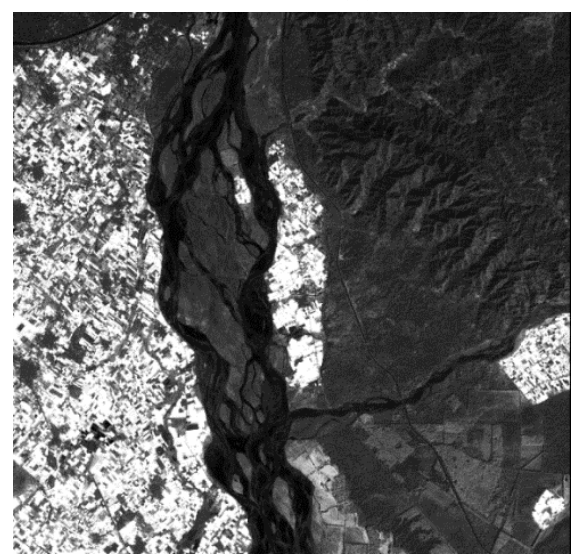

(d)

Fig. 3. (a) Standard FCC of Formosat-2 image (1333 x 1372 pixels), Haridwar Site, Uttarakhand; (b) Classified Image- Wheat Class (PCM), (c) Classified Image- Wheat Class (PCM-S), (d) Classified Image- Wheat Class (PCM-S with ADPLICM)

Table 1. RMSE of algorithms

\begin{tabular}{ll}
\hline Algorithm & RMSE \\
\hline PCM & 0.081 \\
PCM-S & 0.069 \\
PCM -S with ADPLICM & 0.066 \\
\hline
\end{tabular}

\section{Conclusion}

In this paper, a novel PCM-S with ADPLICM algorithm was introduced for image classification and overcome the disadvantages of PCM and PCM-S algorithms. This is achieved by introducing parameter ' $\alpha$ ' and ' $\mathrm{S}_{\text {ir }}$ ' in PCM$S$ with ADPLICM to incorporate local information and to supervise the effect of neighbors terms which makes the 
PCM-S with ADPLICM algorithm able to overcome the limitation of conventional PCM and PCM-S. Experiment was conducted on FORMOSAT-2 multispectral image to demonstrate the performance of PCM-S with ADPLICM algorithm compared to PCM and PCM-S. In case of PCM-S with ADPLICM, RMSE is minimum which means classified image is least effected by noise.

\section{References}

[1] Thomas Lillesand JC, Kiefer RW. Remote sensing and image interpretation. New York: John Willey; 1979.

[2] Bezdek JC, Ehrlich R, Full W. FCM: The fuzzy c-means clustering algorithm. Computers \& Geosciences. 1984;10(2-3):191-203.

[3] Zadeh LA. Fuzzy sets as a basis for a theory of possibility. Fuzzy Sets and Systems. 1978;1(1):3-28.

[4] Krishnapuram R, Keller JM. A possibilistic approach to clustering. IEEE Transactions on Fuzzy Systems. 1993;1(2):98-110.

[5] Krishnapuram R, Keller JM. The possibilistic c-means algorithm: insights and recommendations. IEEE Transactions on Fuzzy Systems. 1996;4(3):385-393.

[6] Wu KL, Yang MS. Alternative c-means clustering algorithms. Pattern Recognition. 2002;35(10):2267-2278.

[7] Li K, Huang HK, Li KL. A modified PCM clustering algorithm. In: Proceedings of the 2003 International Conference on Machine Learning and Cybernetics. IEEE;2003. p. 1174-1179 (Vol. 2).

[8] Kumar A, Ghosh SK, Dadhwal VK. ALCM: Automatic land cover mapping. Journal of the Indian Society of Remote Sensing. 2010;38(2):239-245.

[9] Chawla S. Possibilistic c-means-spatial contextual information based sub-pixel classification approach for multi-spectral data. University of Trento. 2010.

[10] Geman S, Geman D.Stochastic relaxation, gibbs distributions, and the bayesian restoration of images. IEEE Trans. Pattern Anal. Mach. Intell. 1984:PAMI-6(6):721-741.

[11] Derin H, Elliott H. Modeling and segmentation of noisy and textured images using Gibbs random fields. IEEE Transactions on Pattern Analysis \& Machine Intelligence. 1987;9(1):39-55.

[12] Sethi I, Jain A.Artificial neural networks and statistical pattern recognition: old and new connections. Elsevier Science Press; 1991. p. 155-174.

[13] Mahata N, Kahali S, Adhikari SK, Sing JK. Local contextual information and Gaussian function induced fuzzy clustering algorithm for brain MR image segmentation and intensity inhomogeneity estimation. Applied Soft Computing. 2018;68:586-596.

[14] Ahmed MN, Yamany SM, Mohamed N, Farag AA, Moriarty T. A modified fuzzy c-means algorithm for bias field estimation and segmentation of MRI data. IEEE Transactions on Medical Imaging. 2002;21(3):193-199.

[15] Chen S, Zhang D. Robust image segmentation using FCM with spatial constraints based on new kernelinduced distance measure. IEEE Transactions on Systems, Man, and Cybernetics, Part B (Cybernetics). 2004;34(4):1907-1916.

[16] Krinidis S, Chatzis V. A robust fuzzy local information C-means clustering algorithm. IEEE Transactions on Image Processing. 2010;19(5):1328-1337.

[17] Zhang H, Wang Q, Shi W, Hao M. A novel adaptive fuzzy local information c-means clustering algorithm for remotely sensed imagery classification. IEEE Transactions on Geoscience and Remote Sensing. 2017;55(9):5057-5068.

[18] Singh A, Kumar A. Fuzzy based approach to incorporate spatial constraints in possibilistic c-means algorithm for remotely sensed imagery. in: International Conference on Sustainable Computing in Science, Technology \& Management. 20 February 2019.

(C) 2019 by the author(s). This work is licensed under a Creative Commons Attribution 4.0 International License (http://creativecommons.org/licenses/by/4.0/). Authors retain copyright of their work, with first publication rights granted to Tech Reviews Ltd. 